Article

\title{
Effects of a Dielectric Barrier Discharge (DBD) on Characteristics of Polyaniline Nanoparticles Synthesized by a Solution Plasma Process with an Ar Gas Bubble Channel
}

\author{
Jun-Goo Shin ${ }^{1,+}{ }^{,}$Bhum Jae Shin ${ }^{2,+}$, Eun Young Jung ${ }^{1}$, Choon-Sang Park ${ }^{3}$, Jae Young Kim ${ }^{4}$ (i) \\ and Heung-Sik Tae ${ }^{1, *}$ \\ 1 School of Electronics Engineering, College of IT Engineering, Kyungpook National University, \\ Daegu 41566, Korea; bmw345@ee.knu.ac.kr (J.-G.S.); eyjung@knu.ac.kr (E.Y.J.) \\ 2 Department of Electronics Engineering, Sejong University, Seoul 05006, Korea; hahusbj@sejong.ac.kr \\ 3 Department of Electronics and Computer Engineering, College of Engineering, Kansas State University, \\ Manhattan, NY 66506, USA; purplepcs@ksu.edu \\ 4 Department of New Biology, Daegu Gyeongbuk Institute of Science \& Technology, Daegu 42988, Korea; \\ jyk@dgist.ac.kr \\ * Correspondence: hstae@ee.knu.ac.kr; Tel.: +82-53-950-6563 \\ + Jun-Goo Shin and Bhum Jae Shin contributed equally to this work.
}

Received: 6 August 2020; Accepted: 24 August 2020; Published: 27 August 2020

\begin{abstract}
The quality of polyaniline nanoparticles (PANI NPs) synthesized in plasma polymerization depends on the discharge characteristics of a solution plasma process (SPP). In this paper, the low temperature dielectric barrier discharge (DBD) is introduced to minimize the destruction of aniline molecules induced by the direct current (DC) spark discharge. By adopting the new electrode structure coupled with a gas channel, a low temperature DBD is successfully implemented in a SPP, for the first time, thus inducing an effective interaction between the Ar plasma and aniline monomer. We examine the effects of a low temperature DBD on characteristics of polyaniline nanoparticles synthesized by a SPP with an Ar gas bubble channel. As a result, both carbonization of aniline monomer and erosion of the electrode are significantly reduced, which is confirmed by analyses of the synthesized PANI NPs.
\end{abstract}

Keywords: solution plasma; polyaniline nanoparticle; dielectric barrier discharge; polymerization; gas bubble channel

\section{Introduction}

Over the past decades, there has been considerable interest in the synthesis of nanomaterials due to unique electrical, optical, magnetic, and catalytic properties. Among the various methods for nanomaterial synthesis, the solution plasma process (SPP) is a simple and eco-friendly process because a plasma provides reactive chemical species and radicals without any strong chemical reagents [1-3]. However, it is very difficult to generate a discharge in liquid because its density is $10^{4}$ times higher than gas. In general, in a SPP, a discharge is formed in a liquid by applying a high voltage between a pair of pin-type metal electrodes with a narrow inter-electrode distance. Therefore, the strong direct current (DC) spark discharge is generated locally in the metal electrode region, whereby the electrode material is evaporated or sputtered, rapidly cooling in the liquid to form nanoparticles. Accordingly, the SPP has been mainly used to synthesize metal nanoparticles [4-9]. 
Recently, various applications using organic nanoparticles have been intensively studied [10-17]. In particular, polyaniline (PANI) has attracted a significant attention because it is applicable to various promising electronic devices such as supercapacitors, sensors, corrosion protective layers, and flexible displays due to such good features as good environmental and high chemical stability, thermal stability, low cost, and easy synthesis [18-28]. In general, since a strong DC spark discharge is formed in the conventional SPP, it is difficult to synthesize organic nanoparticles requiring low temperature discharge. In a previous study, we introduced the gas channel into the solution to synthesize organic nanoparticles by the SPP method. The pulsed DC streamer discharge within the Ar bubble channel could be stably generated with a significantly reduced firing voltage. It has also been demonstrated that polyaniline nanoparticles (PANI NPs) can be synthesized by SPP [29]. Nevertheless, a strong DC streamer discharge occurred between the two pin-type metal electrodes in the Ar bubble channel, resulting in contamination of the electrode erosion. In particular, the synthesized PANI NPs were observed to have a high carbon content. It is inferred that the Ar plasma energy is high enough to destroy aniline molecules. Therefore, in order to suppress destruction of aniline molecules, low-temperature discharge is essential. The dielectric barrier discharge (DBD) is a low-temperature discharge because the discharge current is limited due to the dielectric barrier, which is suitable to suppress carbonization of aniline monomers and expected to facilitate better properties of PANI NPs synthesized by SPP.

In this paper, we introduced a new electrode structure coupled with a gas channel for DBD and synthesized PANI NPs using DBD for the first time in SPP. The new electrode structure consists of the pin-type electrode in the quartz tube and another cylindrical electrode at the outside quartz tube (hereinafter, 'DBD electrode structure' or 'DBD structure'). The characteristics of DBD in solution are monitored by using a high-speed camera, photo sensor amplifier, and intensified charge coupled device (ICCD). In particular, the radiative species present in the plasma as a result of interactions between the aniline monomer and Ar plasma are monitored by optical emission spectroscopy (OES). To characterize the PANI NPs, Fourier transform infrared (FTIR), field emission scanning electron microscopy (FE-SEM), high resolution transmission electron microscopy (HR-TEM), and X-ray diffraction (XRD) are also examined.

\section{Materials and Methods}

\subsection{Experimental Setup}

The plasma apparatus was made of a glass cylinder with an outer diameter (O.D.), inner diameter (I.D.), and height of 40,34, and $80 \mathrm{~mm}$, respectively. The tungsten electrode was located in the gas bubble channel inside the quartz tube where its diameter was $0.5 \mathrm{~mm}$ and its exact position extruded $1 \mathrm{~mm}$ from the end of a capillary quartz tube. On the other hand, the copper electrode was wrapped on the surface outside the quartz where its width was $5 \mathrm{~mm}$. The copper electrode was equipped $3 \mathrm{~mm}$ away from the end of the capillary quartz tube. The gap between the two capillary quartz tubes was $2 \mathrm{~mm}$. The Ar gas with high purity (99.999\%, Linde Korea, Seoul, Korea) was used as a main gas for producing the plasma and supplied with a flow rate of 100 standard cubic centimeters per minute (sccm) controlled by a mass flow meter (MKS type 1179, range $2000 \mathrm{sccm}$, MKS Instrument Inc., Andover, MA, USA) and was fed through both capillary quartz tubes. The amount of liquid aniline monomer was $23 \mathrm{~mL}$. A bipolar pulse with an amplitude of $8 \mathrm{kV}_{\mathrm{p}-\mathrm{p}}$ and a frequency of $5 \mathrm{kHz}$ was generated by a high voltage amplifier (20/20C-HS, Trek, Inc., Lockport, NY, USA) and a pulse generator (AFG-3102, Tektronix Inc., Beaverton, OR, USA). The bipolar pulse duty ratio was $100 \mu \mathrm{s}$ and the process time for polymerization was $2 \mathrm{~h}$.

\subsection{Synthesis of Polyaniline}

The PANI NPs were synthesized by the SPP with low temperature DBD. The synthesized PANI NPs were mixed with ethanol and purified using the centrifugal separator for $20 \mathrm{~min}$ at 10,000 rpm. Next, the PANI NPs were added to distilled water and rinsed using the centrifugal separator at the 
same condition. This cleaning process was repeated twice. Finally, the solid PANI NPs powder were obtained after being dried at $60^{\circ} \mathrm{C}$ in oven for $12 \mathrm{~h}$. In other words, the solid particles obtained from the plasma processed liquid were used as measurement samples for various analyses.

\subsection{Voltage-Current (V-I) and Electrical Power Measurement}

To examine the dielectric barrier discharge in the liquid state, the discharge voltage was measured by a high voltage probe (P6015A, Tektronix Inc., Beaverton, OR, USA) connected to the powered electrode, i.e., a tungsten electrode, and the corresponding discharge current was measured by a current probe (Model 4100, Pearson Elec. Inc., Palo Alto, CA, USA) connected to the grounded electrode i.e., copper electrode. Electrical power consumption was measured with a digital power meter (WT210, Yokogawa Electric, Tokyo, Japan) for different discharge structures, such as DBD and DC structure. The digital power meter was connected to a power source for measuring the electrical power supplied from the power source to the discharge area.

\subsection{Optical Emission Spectroscopy}

The radiative species present in the plasma as result of interaction between the aniline monomer and Ar plasma were monitored by an optical emission spectrometer (OES) (Ocean Optics USB 4000, response range: 200-1100 nm, optical resolution: 1.5 nm, Ocean Optics Inc., Dunedin, FL, USA) during SPP with low temperature DBD. The OES system consisted of lens (Collimating Lenses, Ocean Optics Inc., Dunedin, FL, USA), optical fiber (P600-20 UV-VIS, range 300 nm-1.1 $\mu \mathrm{m}$, Ocean Optics Inc., Dunedin, FL, USA) and OES. The light focused by the lens from the plasma region is transmitted into the OES through an optical fiber.

\subsection{High Speed Camera}

The evolution of the Ar gas bubble channel was monitored with a high speed camera (Phantom Miro C110, AMETEK, Wayne, NJ, USA). In this case, two lenses (Nikon AF Nikkor 105 mm, 1:2.8 D, and Nikon-AF 36 mm DG, Nikon, Tokyo, Japan) were used at 5000 frames per second (fps) with a shutter time of $200 \mu$ s. The resolution was $272 \times 256$.

\subsection{Intensified Charge-Coupled Device}

The low temperature DBDs during SPP for plasma polymerization were monitored by an intensified charge-coupled device (ICCD) camera (Princeton Instruments, PI-MAX 2) in both shutter modes with exposure times of 5 and $100 \mathrm{~ms}$, respectively.

\subsection{Scanning Electron Microscopy}

The shapes of the synthesized PANI NPs were monitored by field emission-scanning electron microscopy (FE-SEM, SU8220, Hitachi Korea Co. Ltd., Seoul, Korea) with an accelerating voltage and current of $5 \mathrm{kV}$ and $10 \mathrm{~mA}$, respectively.

\subsection{Transmission Electron Microscopy}

High resolution images and selected area electron diffraction (SAED) of synthesized PANI NPs were measured by transmission electron microscopy (TEM). This measurement was conducted with a Titan G2 ChemiSTEM CS Probe (FEI Company, Hillsboro, OR, USA). Samples were dispersed in ethanol and obtained with a carbon-coated copper grid. Energy dispersive X-ray spectroscopy (EDS) (SU8220, Hitachi Korea Co. Ltd., Seoul, Korea) was performed to find elements of synthesized PANI NPs.

\subsection{Fourier Transform Infrared Spectroscopy}

The crystalline phase of the synthesized PANI NPs were measured by Fourier transform infrared spectroscopy (FTIR PerkinElmer, Waltham, MA, USA). The FTIR spectra were measured by averaging 
128 scans at a wavenumber resolution of $0.6 \mathrm{~cm}^{-1}$ in the range from $600 \mathrm{~cm}^{-1}$ to $4000 \mathrm{~cm}^{-1}$ using the attenuated total reflection (ATR) mode.

\subsection{X-ray Diffraction}

In order to analyze the crystalline structure of the synthesized PANI NPs powder, and impurities such as tungsten, the synthesized PANI NPs powder was evaluated by X-Ray diffractometer (XRD, D8 Discover Bruker, USA) at the Korea Basic Science Institute (KBSI, Daegu, Korea). In the XRD analysis, the data were acquired with $2 \theta$ angle ranging from $10^{\circ}$ to $80^{\circ}$ at $0.08^{\circ}$ intervals and $\mathrm{Cu}-\mathrm{k} \alpha$ $(\lambda=1.54 \AA)$ was used as the $\mathrm{X}$-ray source.

\section{Results}

Figure 1a shows the schematic diagram of an experimental apparatus with the proposed DBD electrode structure employed in this study. Since the discharge is very difficult to produce in a solution, especially a liquid aniline monomer with a high dielectric strength, the concept of a bubble channel has been proposed to be able to form a discharge in a gaseous state in a liquid aniline monomer [29]. Figure $1 \mathrm{~b}$ shows a schematic of the DC electrode structure where the pair of pin-type tungsten (W) electrodes face each other in the previous study [29]. When the Ar gas was fed through both quartz tubes, an Ar bubble channel was formed to surround both of the $\mathrm{W}$ electrodes, thereby playing a significant role in providing a gaseous discharge path in liquid aniline. Accordingly, it was observed that a stable pulsed DC streamer occurred along the bubble channel at low applied voltage, which was not a conventional spark discharge in liquid. For effective polymer synthesis, both of the $W$ electrodes were protruded into a liquid aniline to activate a reaction. As a result, the synthesis of PANI NPs using the SPP with the bubble channel was demonstrated. This DC streamer was still strong enough to induce the electrode erosion, including a carbonization. Figure 1c shows the proposed DBD electrode structure with the cylindrical copper $(\mathrm{Cu})$ electrode outside the left quartz tube instead of the pin-type W electrode. Accordingly, the quartz tube $\left(\varepsilon_{\mathrm{r}}=3.78\right)$ acted as a dielectric layer to capacitively limit a discharge current with an application of a bipolar pulse. In particular, the $\mathrm{Cu}$ electrode was $3 \mathrm{~mm}$ away from the end of quartz tube, thus preventing direct connection of the bubble channel between the $\mathrm{W}$ and $\mathrm{Cu}$ electrodes. Nonetheless, a strong DC streamer discharge could be produced in the outside region of the cylindrical $\mathrm{Cu}$ electrode due to charged gas bubbles during the plasma discharge, even under the DBD electrode structure, as shown in Figure 1d. For this reason, the bubble block plate was introduced into the left quartz tube, such that the stable DBD could be obtained thanks to the bubble block plate, as shown in Figure 1e. The bubble block plate was made of Teflon, and its dimensions were a width of $1 \mathrm{~mm}$, a height of $113 \mathrm{~mm}$ and a length of $27 \mathrm{~mm}$. 


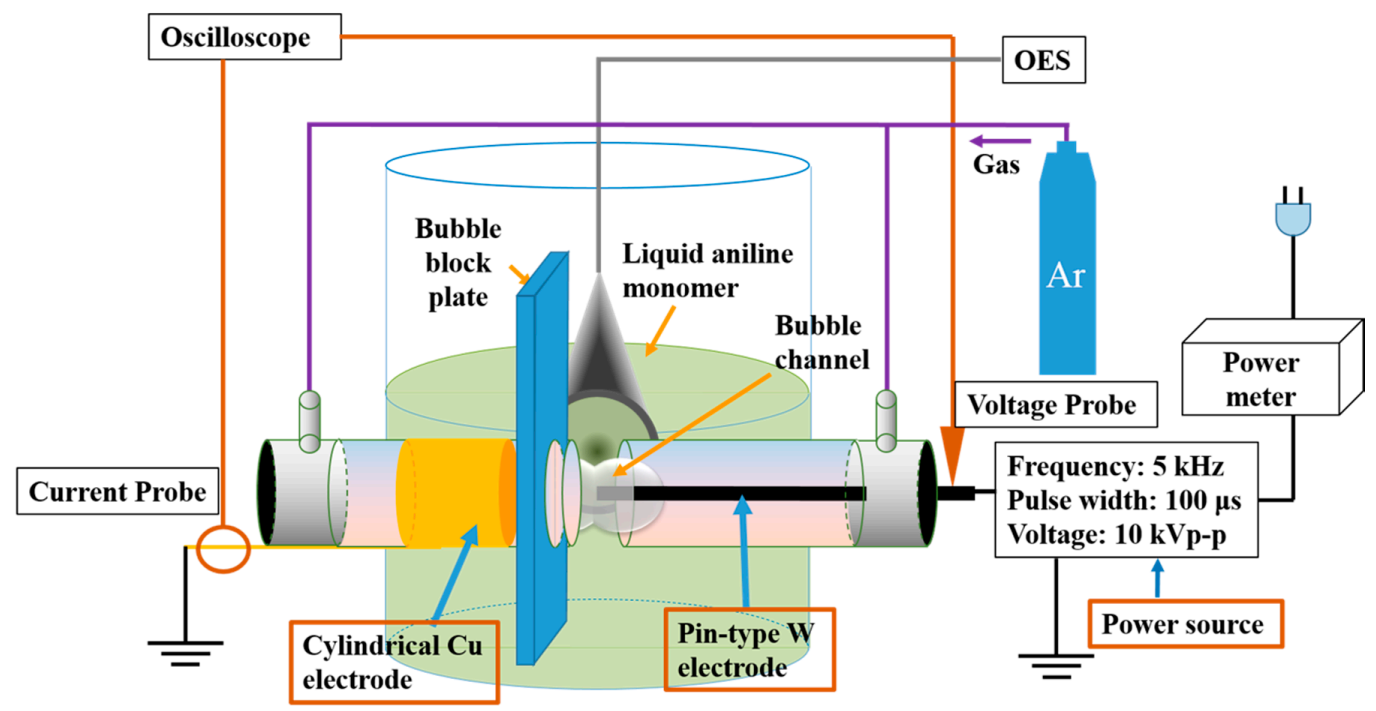

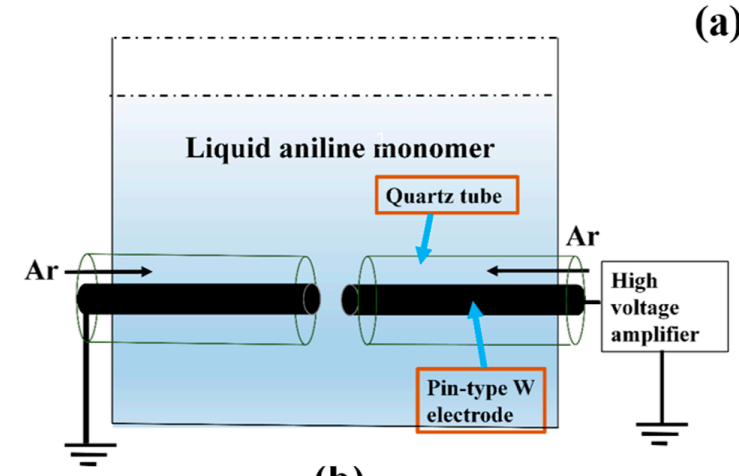

(b)

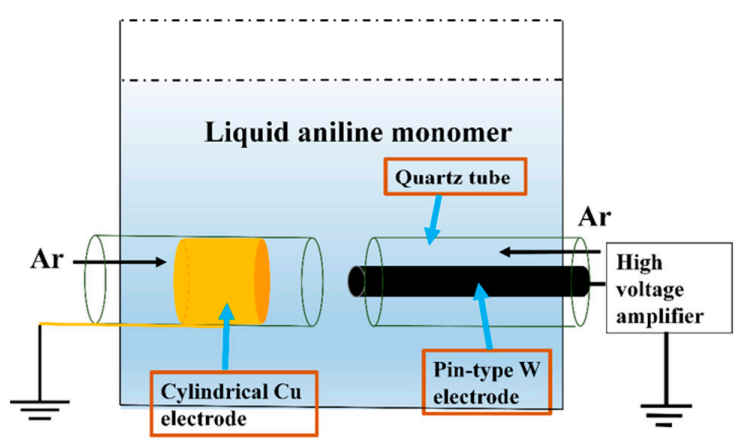

(c)

(a)

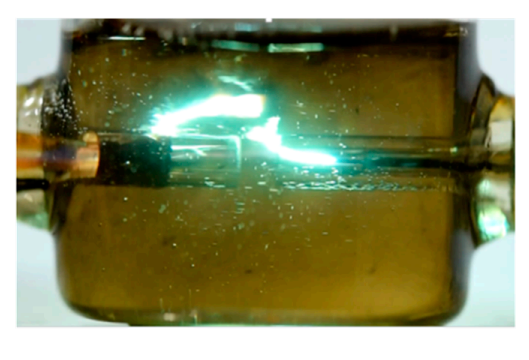

(d)

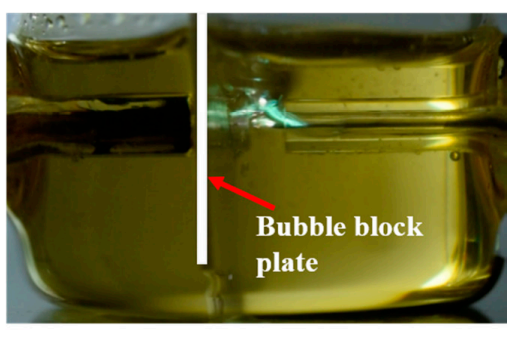

(e)

Figure 1. Experimental setup of the proposed DBD electrode structure with a grounded cylindrical copper electrode and a bubble block plate for plasma polymerization in liquid aniline monomer: (a) experimental setup, (b) DC electrode structure, (c) DBD electrode structure, (d) discharge image under DBD electrode structure without a bubble block plate, and (e) discharge image under DBD electrode structure with a bubble block plate.

Figure 2a shows the temporal evolution of a gas bubble in liquid aniline without discharge, using a high-speed camera. The Ar gas injected from both tubes aggregated around the inlet in a bubble form to combine into a larger bubble, finally forming the gas bubble channel, which played a role in providing the discharge path in liquid aniline monomer between the cylindrical $\mathrm{Cu}$ and pin-type $\mathrm{W}$ electrodes. Then, immediately after forming the bubble channel, the Ar gas bubble was raised upwards due to the buoyancy, as shown in Figure 2a. The discharge was produced repeatedly only while the Ar gas bubble was moved upwards after forming a bubble channel, i.e., during the time period of 
about $19.2 \mathrm{~ms}$ ranging from 12.4 to $31.6 \mathrm{~ms}$. No discharge was observed only under the liquid aniline condition, i.e., when the gas bubble channel was not formed. Accordingly, the discharge could be produced only during a period of gas-bubble channel formation, as shown in Figure 2a. We reported the temporal evolution of discharge formation within the bubble channel by a high-speed camera in the previous DC electrode structure [29]. In this study, however, the intensity of the DBD was too weak to take a discharge image using a high-speed camera, such that it was monitored with an ICCD camera.

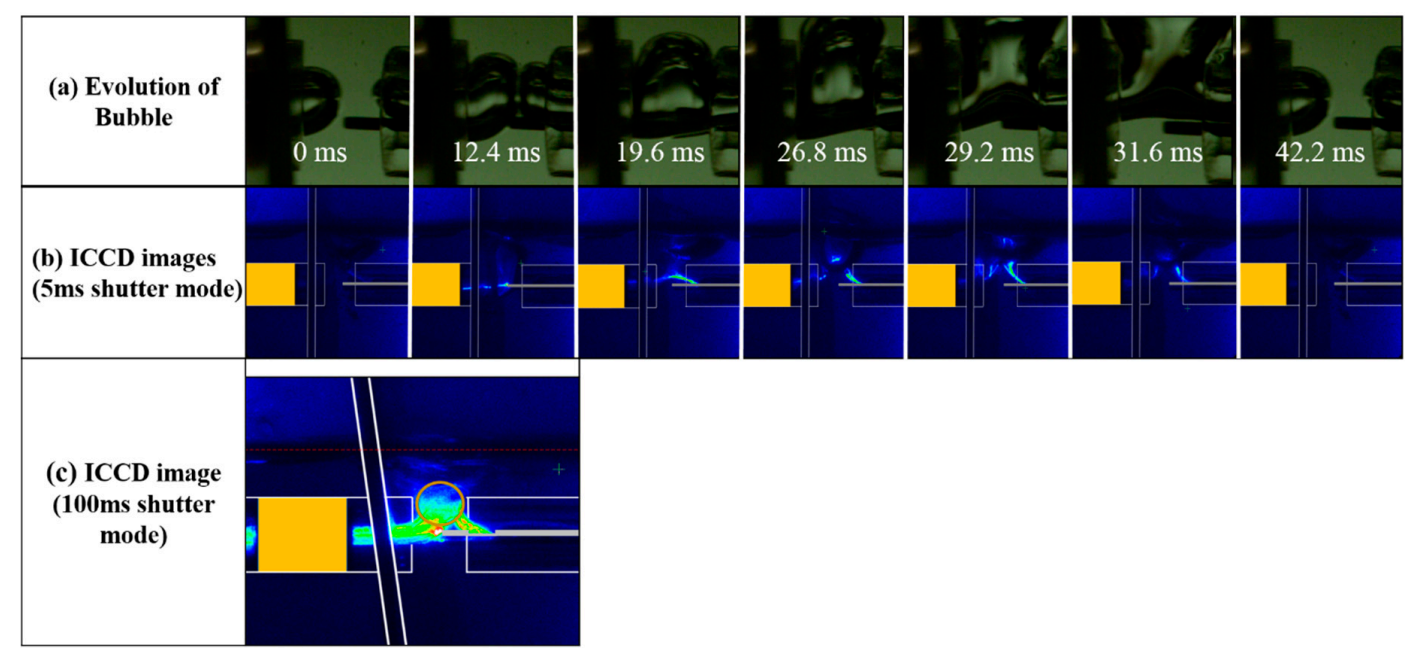

Figure 2. (a) Temporal evolution of the gas bubble in liquid aniline without discharge using a high-speed camera, (b) DBD images taken during exposure time of $5 \mathrm{~ms}$ in the shutter mode of an ICCD, and (c) a DBD image taken during exposure time of $100 \mathrm{~ms}$ in the shutter mode of an ICCD.

Figure $2 \mathrm{~b}$ shows the discharge images taken in a shutter mode with an exposure time of $5 \mathrm{~ms}$ using an ICCD camera. In the previous DC electrode structure, since the electric field was concentrated at the end of the $\mathrm{W}$ electrode, the discharge was mainly generated from the end of the cathode to the upper surface area of the anode along the Ar bubble channel in the discharge region placed between two quartz tubes [29]. In the proposed DBD electrode structure, however, the discharge path of a DBD must be created through the quartz tube under the cylindrical $\mathrm{Cu}$ electrode. Thus, unlike the DC structure in which the electric field was concentrated at the cathode, it was widely distributed inside the quartz tube surrounded by the cylindrical $\mathrm{Cu}$ electrode. As shown in Figure $2 b$, the discharge path was observed to ignite from the $\mathrm{W}$ electrode in the direction of the center axis of the quartz tube under the cylindrical Cu electrode. The DBD was observed only when the gas bubble channel was formed, as shown in Figure 2b.

Figure 2c shows the discharge image taken in a shutter mode with an exposure time of $100 \mathrm{~ms}$, which corresponds to being around two bubble formation cycles. In Figure 2c, the most intense discharge was produced in the vicinity of the pin-type $\mathrm{W}$ electrode, whereas the discharge path was located in the direction of the center axis of the tube in the cylindrical $\mathrm{Cu}$ electrode. Furthermore, the discharge region was observed to be ascended due to the buoyance of the bubble channel. In particular, the active interaction between the Ar channel plasma and liquid aniline monomer for plasma polymerization would mainly occur in the ascended discharge region located in the region of the circle, shown in Figure 2c, where the OES was monitored. In DC discharge, the discharge current was maintained continuously during the application of the voltage [29]. On the contrary, the discharge current in the DBD flowed through the quartz, which essentially acted as a capacitor, charging with the opposite polarity. Therefore, DBD was spontaneously terminated due to the formation of the opposite charges accumulating in the quartz tube. In general, the analysis of instantaneous waveforms was very difficult because the bubble evolution and discharge formation were irregular in liquid aniline. Therefore, the current waveforms were averaged 100 times to analyze the overall trend. 
Figure 3a shows the waveforms of discharge voltage measured during low temperature DBD between the two electrodes. The discharge voltage showed an amplitude of about $8 \mathrm{kV}$ and rising and falling times of around $20 \mu \mathrm{s}$.

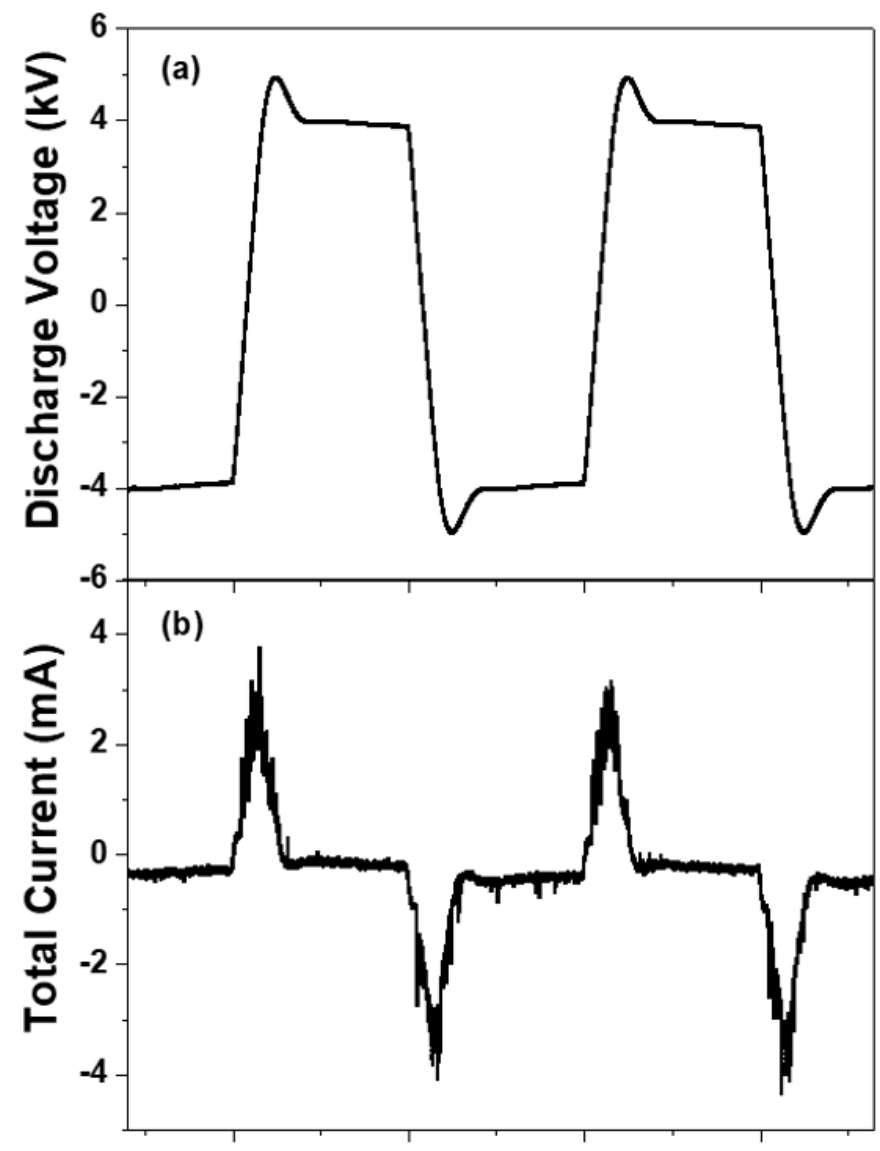

Figure 3. Waveforms of (a) discharge voltage and (b) total current (= discharge current plus displacement current) measured during low temperature DBD between two electrodes.

Figure $3 \mathrm{~b}$ shows the total current (= discharge current plus displacement current) with reproducibility during the low temperature DBD. It should be noted that the discharge currents are observed only during the period when the polarity of the voltage is changed, meaning that the cylindrical $\mathrm{Cu}$ electrode with the quartz tube acted as a capacitor and, as such, the discharge was spontaneously terminated within a few $\mu$ s after the discharge was ignited. In other words, the wall charges accumulating in the dielectric layer located below the cylindrical $\mathrm{Cu}$ electrode, i.e., quartz tube in this experiment, may play a role not only in terminating the current discharge, but also in facilitating the production of the ensuing discharge. Accordingly, unlike the DC discharge currents, the DBD currents flowing only during a very short period within the one voltage pulse would enable a production of low temperature discharge. Electrical power consumptions of DBD and DC structures are measured using digital power meter. As a result, the consumption powers are $260 \mathrm{~W}$ for DBD structure and $560 \mathrm{~W}$ for the DC structure, respectively, implying that the DBD structure consumes a low electrical power during the SPP process.

Figure 4 shows the optical emission spectra (OES) measured during plasma polymerization in the proposed DBD structure compared to the previous DC structure. It notes that the intensity of the DBD structure is much weaker than that of the DC structure due to its weak emission. In general, multiple excited Ar lines ranging from 698 to $854 \mathrm{~nm}$ are observed due to the Ar plasma [30]. Molecular emission peaks of $\mathrm{CN}$ and $\mathrm{C}_{2}$ are observed, which are related to excitation or dissociation of liquid aniline monomer induced due to the impact of electrons. The main $\mathrm{CN}$ peak is $386 \mathrm{~nm}$, while the 
$\mathrm{CN}$ violet system is $359 \mathrm{~nm}$ [31]. The $\mathrm{CN}$ emission spectra refer to the connection of the benzene ring with an amine group. The emission spectra of $C_{2}$ are 471.52, 512 and $561 \mathrm{~nm}$, which are typically involved in swan bands observed in the combustion of hydrocarbons in organic materials [32,33]. It also notes that the emission peak of $C_{2}$ is related to the combination of $C$ or the degradation of $\mathrm{CH}$ and $\mathrm{CN}$. Thus, an increase in the emission peak of $\mathrm{C}_{2}$ implies an increase in the probability of synthesis of carbon nanoparticles from liquid aniline monomers, that is, carbonization. Consequently, as shown in Figure 4, the emission peaks of $C_{2}$ in the DBD structure are observed to be significantly reduced compared to those of $C_{2}$ in the DC structure, indicating that carbonization of liquid aniline monomers could be significantly reduced due to low temperature DBD. Furthermore, in the DBD structure, there is no hydrogen Balmer line of $\mathrm{H}$ corresponding to $656.3 \mathrm{~nm}$ indicating dissociation of liquid aniline monomers [31]. These results confirm that the proposed DBD structure can significantly suppress carbonization.

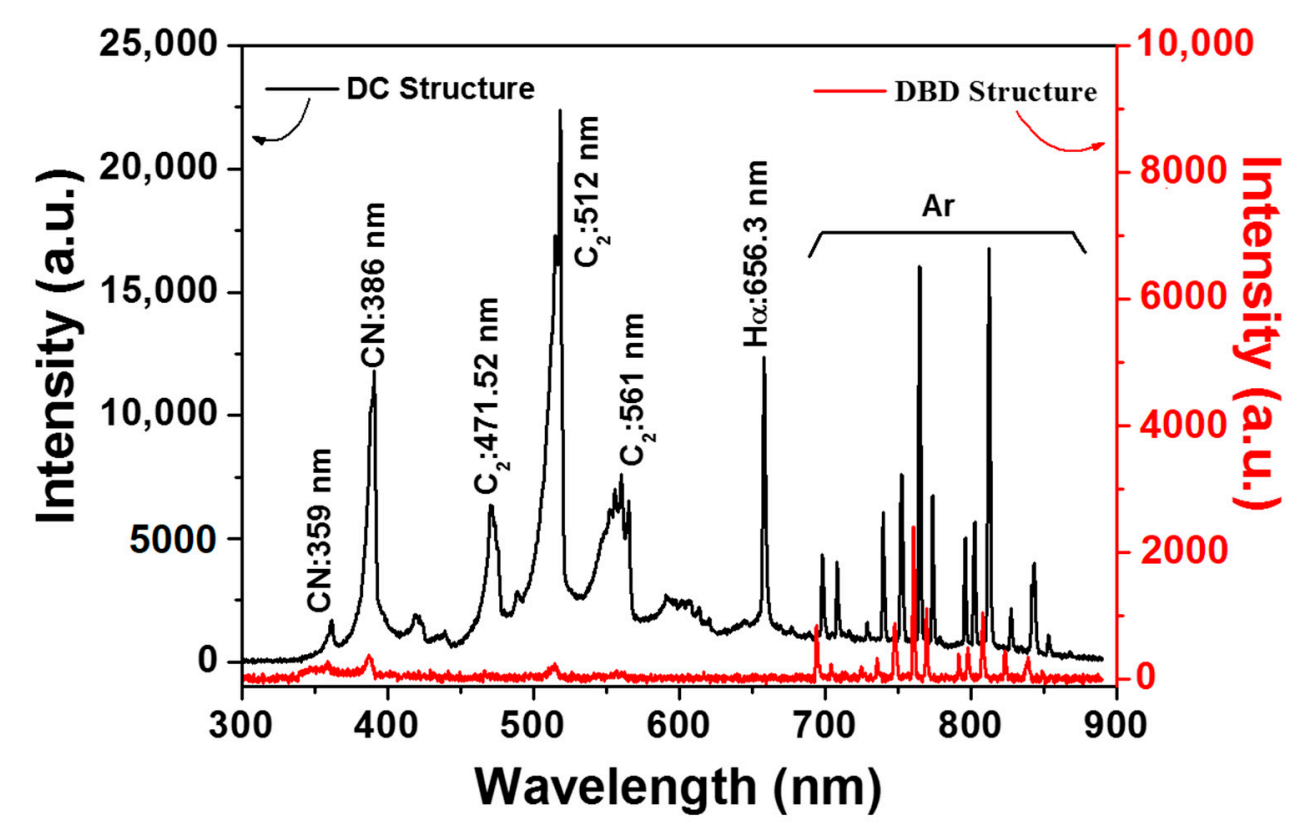

Figure 4. Optical emission spectra (OES) measured during plasma polymerization in the DBD structure compared to the previous DC structure.

In the FTIR spectra of non-processed liquid aniline monomer in Figure 5, peaks of 1493 and $1276 \mathrm{~cm}^{-1}$ are assigned to the $\mathrm{C}=\mathrm{C}$ and $\mathrm{C}-\mathrm{N}$ bonds existing in the non-processed liquid, i.e., liquid aniline monomer. Figure 5 also shows the comparison of FTIR spectra of PANI synthesized in two different electrode structures such as the proposed DBD and previous DC structures. The detailed FTIR peaks of the PANI in Figure 5 are listed in Table 1. The PANI synthesized in the proposed DBD structure was observed to have peaks at $699 \mathrm{~cm}^{-1}$ and $750 \mathrm{~cm}^{-1}$ of $\mathrm{C}-\mathrm{H}$ out of plane bending mode. The peaks of $1248 \mathrm{~cm}^{-1}$ and $1452 \mathrm{~cm}^{-1}$ are assigned to the primary aromatic amine $\mathrm{C}-\mathrm{N}$ bending and $\mathrm{C}=\mathrm{C}$ aromatic ring stretch mode, respectively. The peak at $2959 \mathrm{~cm}^{-1}$ is assigned to the $\mathrm{C}-\mathrm{H}$ asymmetric stretching mode, whereas the peak at $3360 \mathrm{~cm}^{-1}$ is the $\mathrm{N}-\mathrm{H}$ stretching mode. The peaks of $\mathrm{C}-\mathrm{O}$ indicate the bond of alcohol for washing PANI for measurement. The $\mathrm{C}=\mathrm{C}$ and $\mathrm{C}-\mathrm{N}$ bonds corresponding to the peaks of 1493 and $1276 \mathrm{~cm}^{-1}$ in the liquid aniline monomer are considerably reduced in the PANI synthesized by the previous DC structure, but are increased in the PANI synthesized by the proposed DBD structure [34]. 


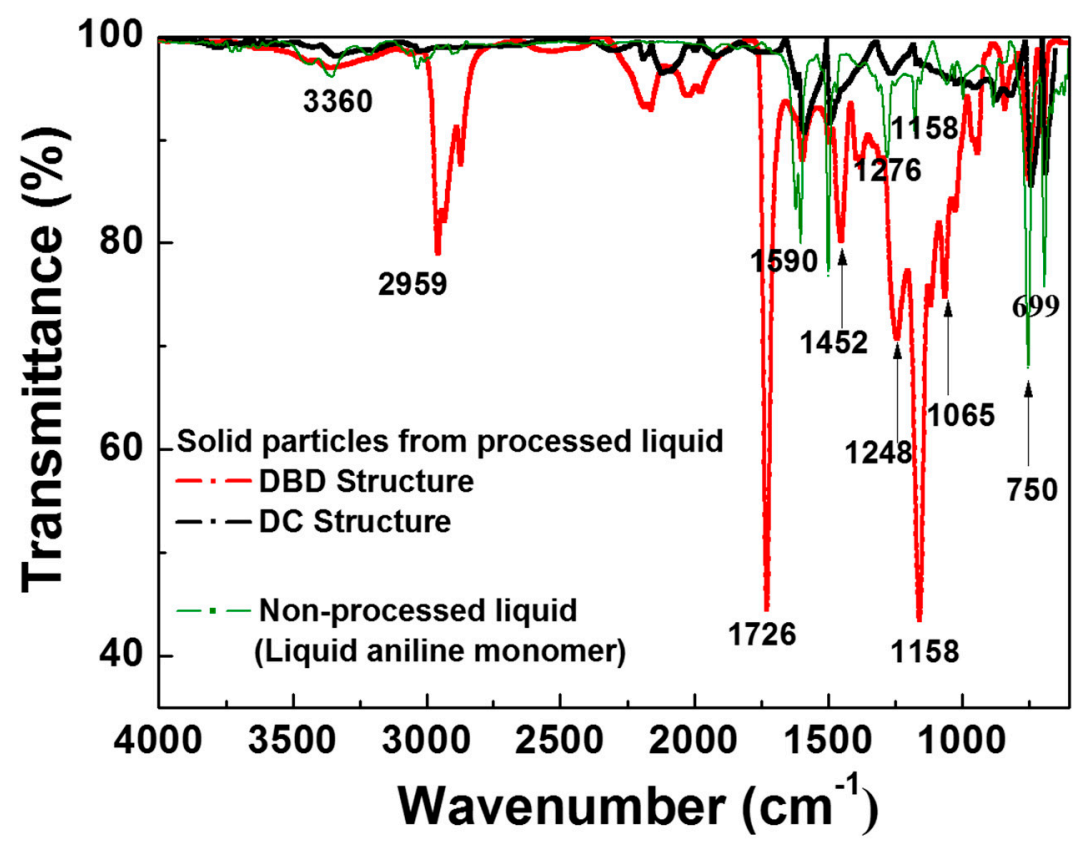

Figure 5. Comparison of FTIR spectra of PANI synthesized in two different discharge electrode structures such as the proposed DBD and previous DC structures, including liquid aniline monomer.

Table 1. FTIR peaks of PANI synthesized in both DBD and DC structures.

\begin{tabular}{cc}
\hline Wavenumber & Vibration Mode \\
\hline $699 \mathrm{~cm}^{-1}$ & $\mathrm{C}-\mathrm{H}$ out of plane bending \\
$750 \mathrm{~cm}^{-1}$ & $\mathrm{C}-\mathrm{H}$ out of plane bending \\
$1065 \mathrm{~cm}^{-1}$ & $\mathrm{C}-\mathrm{O}$ stretching \\
$1158 \mathrm{~cm}^{-1}$ & $\mathrm{C}-\mathrm{O}$ stretching \\
$1248 \mathrm{~cm}^{-1}$ & $\mathrm{C}-\mathrm{N}$ bending \\
$1452 \mathrm{~cm}^{-1}$ & $\mathrm{C}=\mathrm{C}$ ring stretching \\
$1726 \mathrm{~cm}^{-1}$ & $\mathrm{C}-\mathrm{O}$ stretching \\
$2959 \mathrm{~cm}^{-1}$ & $\mathrm{C}-\mathrm{H}$ asymmetric stretching \\
$3360 \mathrm{~cm}^{-1}$ & $\mathrm{~N}-\mathrm{H}$ stretching \\
\hline
\end{tabular}

Most of measured peaks of the PANI synthesized by the proposed DBD structure are more intense than those of the PANI synthesized by the previous DC structure, meaning that an amount of chemical bonds relative to PANI increase due to the low temperature discharge growth condition under the DBD structure. In the N-H peak intensities at $3360 \mathrm{~cm}^{-1}$, both electrode conditions show a slight difference. For the DBD case, however, the peak of $1248 \mathrm{~cm}^{-1}$ is prominent, implying that the PANI has lots of $\mathrm{C}-\mathrm{N}$ bonds, which are related to an increase in the bonds of the benzenoid and quinoid rings.

Figure $6 \mathrm{a}, \mathrm{b}$ shows the FE-SEM images of the PANI nanoparticles synthesized for $3 \mathrm{~h}$ in the proposed DBD and previous DC structures, respectively [29]. As can be seen in Figure 6a, the sizes of the PANI nanoparticles are observed to increase significantly compared to those of the DC structure shown in Figure $6 \mathrm{~b}$. In addition, it is confirmed that the nanoparticles having a wide variety of sizes are synthesized in the proposed DBD structure. In the previous DC structure, as shown in Figure $6 \mathrm{~b}$, the synthesized PANI nanoparticles show a tiny spherical-type nano size structure. Average sizes of PANI nanoparticles for the DBD and DC structures are $277 \mathrm{~nm}$ and $28 \mathrm{~nm}$. It can be inferred that fragmentation of the aniline monomer occurs severely due to the strong DC discharge, resulting in the production of many carbonization reactants as well as PANI nanoparticles. It should be addressed that the PANI nanoparticles were effectively synthesized under the low temperature DBD. In addition, it means that the characteristics of the PANI nanoparticles can be controlled according to the discharge characteristics. 

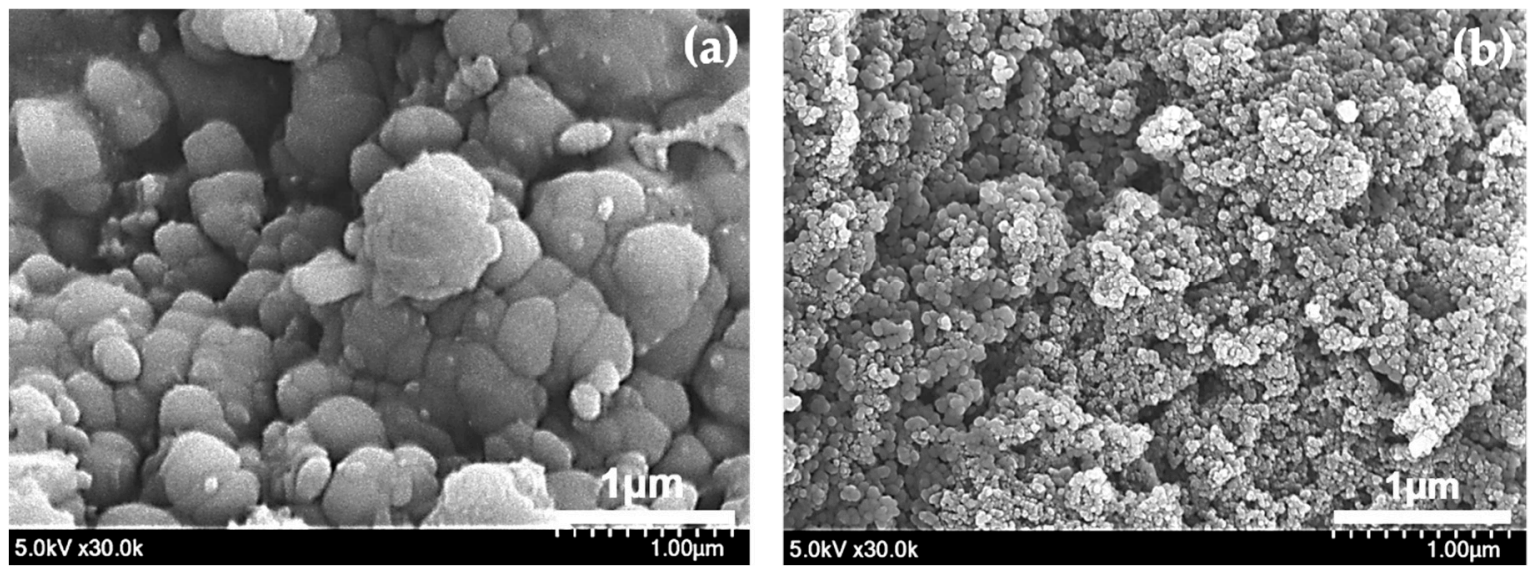

Figure 6. FE-SEM images of PANI nanoparticles synthesized in two different discharge electrode structures; (a) proposed DBD structure and (b) previous DC structure.

Figure 7a,b shows the HR-TEM image and magnified HR-TEM image of PANI synthesized by the proposed DBD structure. EDS analysis in the inset of Figure 7a shows that synthesized PANI includes carbon and nitrogen elements but little tungsten element. It indicates that the erosion of the W electrode is significantly reduced due to the low temperature discharge under the DBD structure. As can be seen in Figure 7b, the specific ordered structure in PANI is not discovered and, as such, the SAED pattern indicates an amorphous pattern. The proposed DBD structure shows the possibility of synthesizing polymer nanoparticles effectively due to the low temperature DBD.
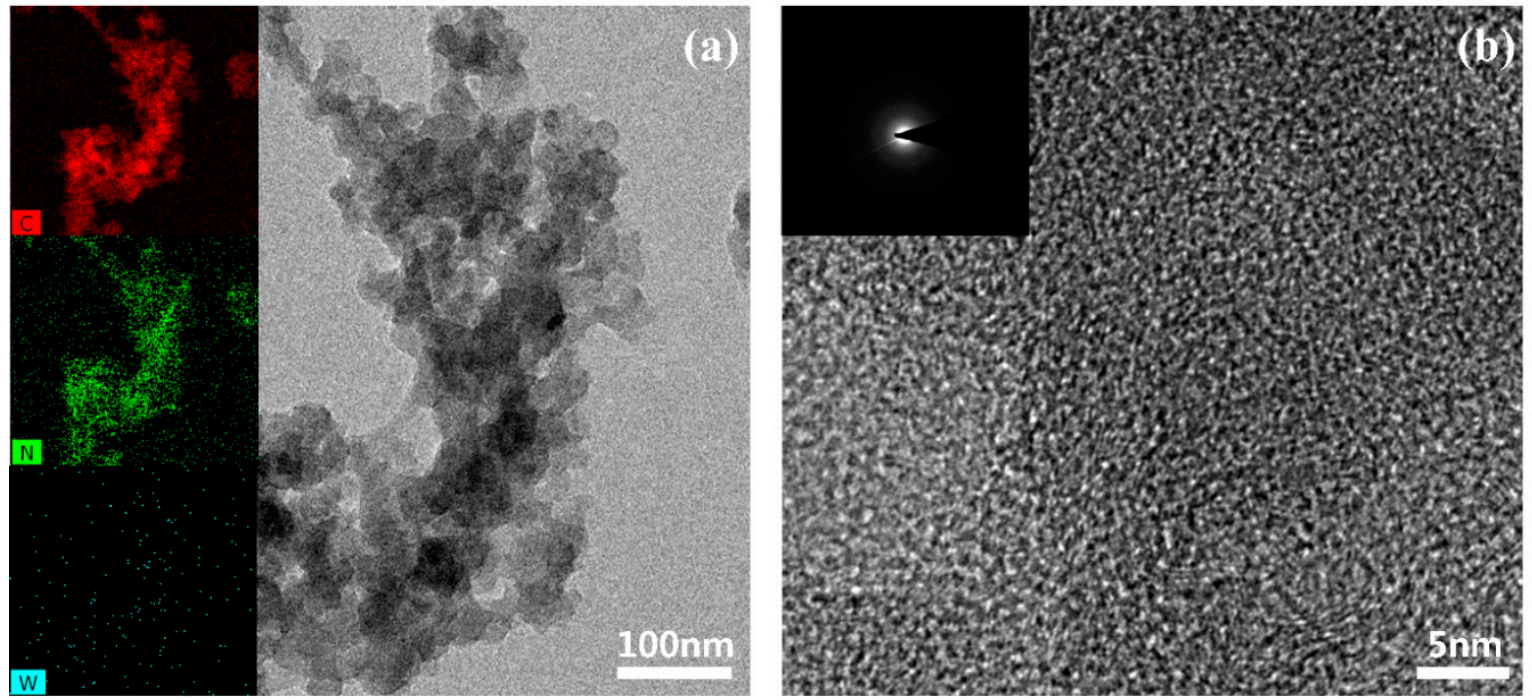

Figure 7. HR-TEM images, SAED pattern and EDS of synthesized PANI by the proposed DBD structure $(\mathbf{a}, \mathbf{b})$ with different magnifications of HR-TEM images; the inset of (a) is carbon, nitrogen and tungsten elements measured by EDS, and the inset of $(\mathbf{b})$ is the SAED pattern.

Figure 8a,b shows the XRD patterns of PANI synthesized in two different discharge electrode structures such as the proposed DBD and previous DC structures, respectively. In the proposed DBD structure of Figure $8 \mathrm{a}$, the intensive XRD peak was detected at $20.07^{\circ}$, corresponding to the (020) crystalligrphic plane, whereas in the previous DC structure, intensive XRD peaks were detected at $20.28^{\circ}$ and $24.92^{\circ}$, corresponding to the (020) and (200) crystalligraphic planes, respectively [35]. Both peaks corresponding to PANI NPs are broad, meaning that these structures are amorphous, but the shape of the XRD pattern in the proposed DBD structure is narrower than in the previous DC structure. In addition, in order to investigate the degree of crystalline for the PANI NPs synthesized 
by the DBD and DC strucutres, we calculated the values of the full width at half maximum (FWHM) and crystalline size using the Scherrer equation [36] based on the XRD data of Figure 8. The resultant values of both FWHM and crystalline size are given in Table 2. In Table 2, the values of FWHM for (202) crystallographic plane reflections were $5.05^{\circ}$ for the DBD structure and $7.15^{\circ}$ for the DC structure, respectively. This result indicates the increase in crystalline degree for the PANI NPs synthesized in the DBD structure. In addition, the peaks of tungsten carbide (WC) were observed only at $43^{\circ}$, corresponding to the (200) crystallographic plane's refections of WC in the proposed DBD structure, whereas various peaks of tungsten carbide were observed at $36.77^{\circ}, 42.6^{\circ}, 61.92^{\circ}, 74^{\circ}$ and $78^{\circ}$, corresponding to the (111), (200), (220), (311) and (222) crystallographic planes of WC in the previous DC structure $[5,37,38]$. These results confirm that the erosion of the tungsten electrode is considerably reduced due to the low temperature discharge under the proposed DBD structure.
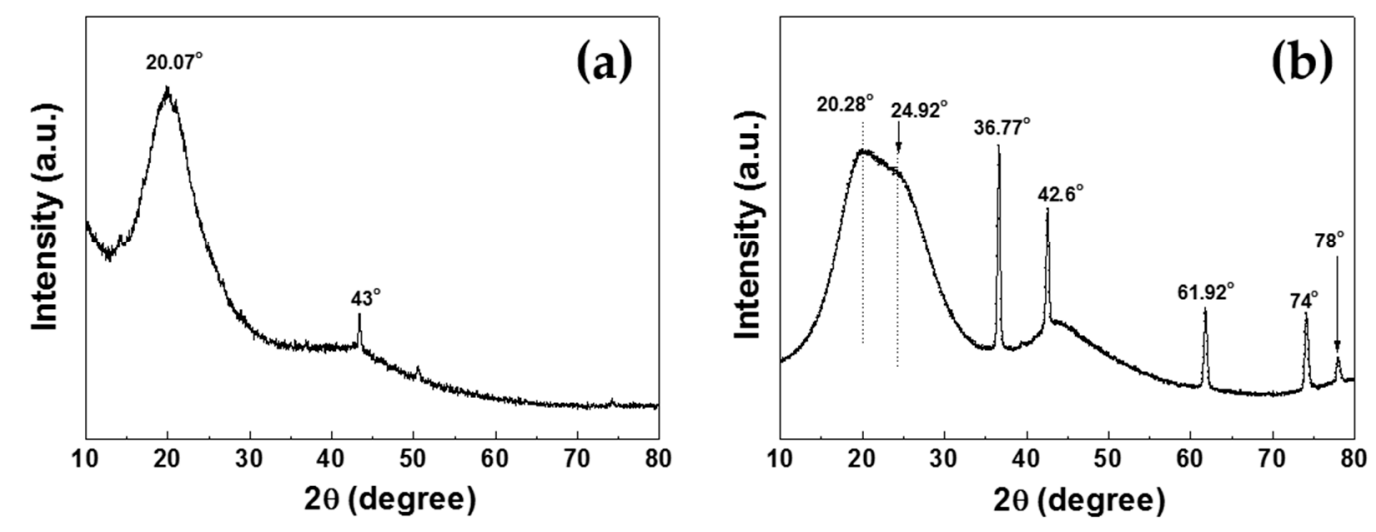

Figure 8. XRD patterns of PANI synthesized in two different discharge electrode structures, such as (a) proposed DBD and (b) previous DC structures.

Table 2. Full width at half maximum (FWHM), crystalline size of PANI nanoparticle powders synthesized in two different discharge structures, DBD and DC structures.

\begin{tabular}{ccccc}
\hline Electrode Strucure & Lattice Diffraction & $\mathbf{2 \theta}$ (Degree) & FWHM (Degree) & Crystalline Size (nm) \\
\hline DBD structure & 020 & $20.07^{\circ}$ & $5.05^{\circ}$ & 1.58 \\
DC structure & 020 & $20.08^{\circ}$ & $7.15^{\circ}$ & 1.11 \\
& 200 & $24.92^{\circ}$ & $8.15^{\circ}$ & 0.98 \\
\hline
\end{tabular}

\section{Conclusions}

This paper investigates the effects of the dielectric barrier discharge (DBD) on the characteristics of PANI NPs synthesized by a SPP with an Ar gas bubble channel. By adopting a new electrode structure featuring the cylindrical $\mathrm{Cu}$ electrode of an external quartz tube with a bubble block plate, a low temperature DBD is produced through an Ar gas bubble channel in liquid aniline monomers for synthesizing PANI NPs. As a result, PANI NPs are successfully synthesized under the low-temperature DBD in liquid aniline monomers. The evolution of the DBD is closely related to the upward movement of the bubble channel, and the active interaction between the Ar channel plasma and the liquid aniline monomer for plasma polymerization would occur mainly in the ascended discharge zone, which is monitored by the ICCD. As shown by the FTIR, PANI NPs synthesized by DBD have lots of C-N bonds, implying the increased bonds of benzenoid and quinoid rings and reduced destruction of liquid aniline monomers. Accordingly, it is observed that the carbon contents, as well as the erosion of the W electrode, can be significantly reduced due to the low temperature DBD. Furthermore, FE-SEM reveals that the average size of PANI NPs grown in the DBD structure is significantly larger than in the previous DC structure. In conclusion, it has been demonstrated that, by applying the proposed DBD electrode structure to the synthesis of PANI NPs using a solution plasma process with an Ar gas bubble channel, the carbonization of aniline monomers and the erosion of electrodes can be significantly reduced. It is 
expected that the adoption of the proposed DBD electrode structure in a solution plasma process with a gas bubble channel can contribute to improving the quality of the synthesized nanoparticles, especially organic nanoparticles.

Author Contributions: J.-G.S., B.J.S. and H.-S.T. conceived and designed the study; J.-G.S. and B.J.S. performed the experiments; J.-G.S., B.J.S., E.Y.J. and J.Y.K. helped to conduct the plasma setup; J.-G.S., B.J.S., E.Y.J., C.-S.P. and H.-S.T. analyzed the data; J.-G.S., B.J.S. and H.-S.T. wrote the majority of the paper. All authors have read and agreed to the published version of the manuscript.

Funding: This research was funded by a National Research Foundation of Korea (NRF) grant funded by the Korean government (MOE) (No. 2020R11A3071693).

Acknowledgments: In this section, the authors would like to thank Sang-Geul Lee at the Korea Basic Science Institute (Daegu) for useful discussion and providing the XRD data.

Conflicts of Interest: The authors declare no conflict of interest.

\section{References}

1. Morishita, T.; Ueno, T.; Panomsuwan, G.; Hieda, J.; Yoshida, A.; Bratescu, M.A.; Saito, N. Fast formation routes of nanocarbons in solution plasma processes. Sci. Rep. 2016, 6, 36880. [CrossRef] [PubMed]

2. Hyun, K.; Saito, N. The solution plasma process for heteroatom-carbon nanosheets: The role of precursors. Sci. Rep. 2017, 7, 3825. [CrossRef] [PubMed]

3. Saito, N.; Bratescu, M.A.; Hashimi, K. Solution plasma: A new reaction field for nanomaterials synthesis. Jpn. J. Appl. Phys. 2018, 57, 0102A4. [CrossRef]

4. Čempel, D.; Nguyen, M.T.; Ishida, Y.; Tsukamoto, H.; Shirai, H.; Wang, Y.; Wu, K.C.-W.; Yonezwa, T. $\mathrm{Au}$ Nanoparticles Prepared Using a Coated Electrode in Plasma-in-Liquid Process: Effect of the Solution pH. J. Nanosci. Nanotechnol. 2016, 16, 9257-9262. [CrossRef]

5. Kim, D.-W.; Li, O.L.; Pootawang, P.; Saito, N. Solution plasma synthesis process of tungsten carbide on N-doped carbon nanocomposite with enhanced catalytic ORR activity and durability. RSC Adv. 2014, 4, 16813-16819. [CrossRef]

6. Pootawang, P.; Saito, N.; Takai, O.; Lee, S.-Y. Synthesis and characteristics of Ag/Pt bimetallic nanocomposites by arc-discharge solution plasma processing. Nanotechnology 2012, 23, 395602. [CrossRef]

7. Pootawang, P.; Saito, N.; Lee, S.-Y. Discharge time dependence of a solution plasma process for colloidal copper nanoparticle synthesis and particle characteristics. Nanotechnology 2013, 24, 055604. [CrossRef]

8. Saito, G.; Sakaguchi, N. Solution plasma synthesis of Si nanoparticles. Nanotechnology 2015, 26, 235602. [CrossRef] [PubMed]

9. Watthanaphanit, A.; Panomsuwan, G.; Saito, N. A novel one-step synthesis of gold nanoparticles in an alginate gel matrix by solution plasma sputtering. RSC Adv. 2014, 4, 1622-1629. [CrossRef]

10. Bandgar, D.K.; Khuspe, G.D.; Pawar, R.C.; Lee, C.S.; Patil, V.B. Facile and novel route for preparation of nanostructured polyaniline (PANi) thin films. Appl. Nanosci. 2014, 4, 27-36. [CrossRef]

11. Lakshmi, G.B.V.S.; Dhillon, A.; Siddiqui, A.M.; Zulfequar, M.; Avasthi, D.K. RF-plasma polymerization and characterization of polyaniline. Eur. Polym. J. 2009, 45, 2873-2877. [CrossRef]

12. Zhang, X.; Zhu, J.; Haldolaarachchige, N.; Ryu, J.; Young, D.P.; Wei, S.; Guo, Z. Synthetic process engineered polyaniline nanostructures with tunable morphology and physical properties. Polymer 2012, 53, 2109-2120. [CrossRef]

13. Valderrama-García, B.X.; Rodríguez-Alba, E.; Morales-Espinoza, E.G.; Chane-Ching, K.M.; Rivera, E. Synthesis and characterization of novel polythiophenes containing pyrene chromophores: Thermal, optical and electrochemical properties. Molecules 2016, 21, 172. [CrossRef]

14. Wang, F.; Li, M.; Wang, B.; Zhang, J.; Cheng, Y.; Liu, L.; Lu, F.; Wang, S. Synthesis and Characterization of Water-Soluble Polythiophene Derivatives for Cell Imaging. Sci. Rep. 2014, 5, 7617. [CrossRef] [PubMed]

15. Rahaman, M.; Aldalbahi, A.; Almoiqli, M.; Alzahly, S. Chemical and Electrochemical Synthesis of Polypyrrole Using Carrageenan as a Dopant: Polypyrrole/Multi-Walled Carbon Nanotube Nanocomposites. Polymers 2018, 10, 632. [CrossRef]

16. Kojabad, Z.D.; Shojaosadati, S.A. Chemical synthesis of polypyrrole nanostructures: Optimization and applications for neural microelectrodes. Mater. Des. 2016, 96, 378-384. [CrossRef] 
17. Wen, J.; Tian, T.; Mei, Z.; Wu, W.; Tian, Y. Synthesis of polypyrrole nanoparticles and their applications in electrically conductive adhesives for improving conductivity. RSC Adv. 2017, 7, 53219-53225. [CrossRef]

18. Baker, C.O.; Huang, X.; Nelson, W.; Kaner, R.B. Polyaniline nanofibers: Broadening applications for conducting polymers. Chem. Soc. Rev. 2017, 46, 1510-1525. [CrossRef]

19. Park, C.-S.; Kim, D.H.; Shin, B.J.; Tae, H.-S. Synthesis and characterization of nanofibrous polyaniline thin film prepared by novel atmospheric pressure plasma polymerization technique. Materials 2016, 9, 39. [CrossRef]

20. Park, C.-S.; Jung, E.Y.; Kim, D.H.; Cho, B.-G.; Shin, B.J.; Tae, H.-S. TOF-SIMS study on nano size conducting polymer prepared by simple atmospheric pressure plasma polymerization technique for display applications. Mol. Cryst. Liq. Cryst. 2017, 651, 16-25. [CrossRef]

21. Park, C.-S.; Kim, D.Y.; Kim, D.H.; Lee, H.-K.; Shin, B.J.; Tae, H.-S. Humidity-independent conducting polyaniline films synthesized using advanced atmospheric pressure plasma polymerization with in-situ iodine doping. Appl. Phys. Lett. 2017, 110, 033502. [CrossRef]

22. Park, C.-S.; Jung, E.Y.; Kim, D.H.; Kim, D.Y.; Lee, H.-K.; Shin, B.J.; Lee, D.H.; Tae, H.-S. Atmospheric Pressure Plasma Polymerization Synthesis and Characterization of Polyaniline Films Doped with and without Iodine. Materials 2017, 10, 1272. [CrossRef] [PubMed]

23. Chaudhary, V.; Kaur, A. Enhanced room temperature sulfur dioxide sensing behaviour of in situ polymerized polyaniline-tungsten oxide nanocomposite possessing honeycomb morphology. RSC Adv. 2015, 5, 73535-73544. [CrossRef]

24. Yang, C.; Zhang, L.; Hu, N.; Yang, Z.; Su, Y.; Xu, S.; Li, M.; Yao, L.; Hong, M.; Zhang, Y. Rational design of sandwiched polyaniline nanotube/layered graphene/polyaniline nanotube papers for high-volumetric supercapacitors. Chem. Eng. J. 2017, 309, 89-97. [CrossRef]

25. Boeva, Z.A.; Sergeyev, V.G. Polyaniline: Synthesis, properties, and application. Polym. Sci. Ser. C 2014, 56, 144-153. [CrossRef]

26. Fratoddi, I.; Venditti, I.; Cametti, C.; Russo, M.V. Chemiresistive polyaniline-based gas sensors: A mini review. Sens. Actuators B Chem. 2015, 220, 534-548. [CrossRef]

27. Deng, J.; Wang, X.; Guo, J.; Liu, P. Effect of the Oxidant/Monomer Ratio and the Washing Post-Treatment on Electrochemical Properties of Conductive Polymers. Ind. Eng. Chem. Res. 2014, 53, 13680-13689. [CrossRef]

28. Abdolahi, A.; Hamzah, E.; Ibrahim, Z.; Hashim, S. Synthesis of Uniform Polyaniline Nanofibers through Interfacial Polymerization. Materials 2012, 5, 1487-1498. [CrossRef]

29. Shin, J.-G.; Park, C.-S.; Jung, E.-Y.; Shin, B.J.; Tae, H.S. Synthesis of a Polyaniline Nanoparticle Using a Solution Plasma Process with an Ar Gas Bubble Channel. Polymers 2019, 11, 105. [CrossRef]

30. Shao, T.; Zhang, C.; Wang, R.; Zhou, Y.; Xie, Q.; Fang, Z. Comparison of atmospheric-pressure He and Ar plasma jets driven by microsecond pulses. IEEE Trans. Plasma Sci. 2015, 43, 726-732. [CrossRef]

31. Ndiaye, A.A.; Lacoste, A.; Bès, A.; Zaitsev, A.; Poncin-Epaillard, F.; Debarnot, D. A Better Understanding of the Very Low-Pressure Plasma Polymerization of Aniline by Optical Emission Spectroscopy Analysis. Plasma Chem. Plasma Process. 2018, 38, 887-902. [CrossRef]

32. Abdul Kalam, S.; Linga Muthy, N.; Mathi, P.; Kommu, N.; Singh, A.K. Correlation of molecular, atomic emissions with detonation parameters in femtoseconf and nanosecond LIBS plasma of high energy materials. J. Anal. At. Spectrom. 2017, 32, 1535-1546. [CrossRef]

33. Zhou, R.; Zhou, R.; Zhang, X.; Fang, Z.; Wang, X.; Speight, R.; Wang, H.; Doherty, W.; Cullen, P.J.; Ostikov, K.K.; et al. High-performance plasma-enable biorefinery of microalgae into value-added products. ChemSusChem 2019, 12, 4976-4985. [CrossRef] [PubMed]

34. Zhu, H.; Peng, S.; Jiang, W. Electrochemical properties of PANI as single electrode of electrochemical capacitors in acid electrolytes. Sci. World J. 2013, 6, 2013-940153. [CrossRef] [PubMed]

35. Zhou, Z.; Zhang, X.; Lu, C.; Yuan, G. Polyaniline-decorated cellulose aerosol nanocomposite with strong interfacial adhesion and enhanced photocatalytic activity. RSC Adv. 2014, 4, 8966-8972. [CrossRef]

36. Du, X.; Xu, Y.; Xiong, L.; Zhu, J.; Mao, S. Polyaniline with high crystallinity degree: Synthesis, structure, and electrochemical properties. J. Appl. Polym. Sci. 2014, 131, 40827. [CrossRef] 
37. Hyun, K.; Ueno, T.; Saito, N. Synthesis of nitrogen-containing carbon by solution plasma in aniline with high-repetition frequency discharges. Jpn. J. Appl. Phys. 2016, 55, 01AE18. [CrossRef]

38. Bafandeh, N.; Mojtahedzadeh, M.; Shafiekhani, A.; Hantehzadeh, M.R.; Sheikh, N. Synthesis of polyaniline films: Case study on post gamma irradiation dose. J. Mater. Sci. Mater. Electron 2016, 27, 10566-10572. [CrossRef] 